\title{
Evolution of age specific dispersal: a general discussion from the standpoint of the invasion fitness concept
}

\author{
Karin Johst and Roland Brandl
}

Johst, K. and Brandl, R. 2000. Evolution of age specific dispersal: a general discussion from the standpoint of the invasion fitness concept. - Web Ecol. 1: 82-85.

\begin{abstract}
The age of dispersers is one of the important factors influencing the ecology and evolution of dispersal. Explanations in textbooks assume special social or genetic advantages to predict the dispersing age class. However, recent theoretical results suggest that age specific dispersal can evolve even without special advantages for the dispersing age class. Here we discuss and interpret these results on the basis of the invasion fitness concept and show with general arguments that the evolution of age specific dispersal is possible for purely demographic reasons. This furthers our general understanding of the evolution of age specific dispersal.
\end{abstract}

K. Johst (kajo@oesa.ufz.de), Centre for Environmental Research Leipzig-Halle Ltd., Dept of Ecological Modelling, Permoserstr. 15, D-04318 Leipzig, Germany. - R. Brandl, Centre for Environmental Research Leipzig-Halle Ltd., Dept of Community Ecology, Theodor-Lieser Str. 4, D-06120 Halle, Germany.

In vertebrate populations dispersal is often biased towards juveniles (Greenwood and Harvey 1982, Cockburn 1992). Therefore, vertebrate ecologists divide dispersal into natal and breeding dispersal to characterise the age of dispersers. Natal dispersal describes the dispersal of juveniles from the site of birth to that of first potential reproduction, whereas the movement of adults between sites is termed breeding dispersal (Greenwood and Harvey 1982). Despite the importance of age specific dispersal in nature, age specific dispersal has been ignored in most of the evolutionary theory of dispersal.

In a previous paper we used a simulation model to study the evolutionary competition of phenotypes which differed not only in their propensity to disperse but also in the age class where dispersal occurred (Johst and Brandl 1999). In our model system natal dispersal could evolve even without assuming special social or genetic advantages for dispersing juveniles. We showed that competitive differences between natal and breeding dispersal can be generated by the age structure of the considered population. These results could be explained by basic evolutionary principles of dispersal biology. For example, a temporally fluctuating environment selects generally for high dispersal (e.g. Johnson and Gaines 1990, McPeek and Holt 1992). Consequently, in such an environment a high dispersal probability has evolved in our simulation model in the most common age class or across several age classes.

In the following we will show that these findings can be understood within the concept of invasion fitness as well. This concept is a novel and powerful tool to predict adaptive dynamics and thus long-term consequences of evolution (Metz et al. 1996, Dieckman 1997, Geritz et al. 1997). The invasion fitness exponent is the central quantity within this concept. 


\section{Invasion fitness exponent}

The invasion fitness exponent $s$ (also called selectivity or long-term growth rate) is defined as the logarithm of the geometric average of the realised fitness values $R(E(t))$ of a small mutant population which tries to invade in an environment $\mathrm{E}$. This environment consists of abiotic and biotic components (Metz et al. 1992, Rand et al. 1994):

$$
s=\lim _{\mathrm{T} \rightarrow \infty} \frac{1}{\mathrm{~T}} \ln \prod_{\mathrm{t}=0}^{\mathrm{T}-1} \mathrm{R}(\mathrm{E}(\mathrm{t}))
$$

The realised fitness values $\mathrm{R}(\mathrm{E}(\mathrm{t}))$ determine the population growth of rare mutants during the course of time $\mathrm{N}(\mathrm{t}+1)=\mathrm{R}(\mathrm{E}(\mathrm{t})) \mathrm{N}(\mathrm{t})$. The abiotic environment comprises the spatial and temporal variability of the resources. The biotic environment is formed by the resident phenotypes and is therefore influenced by the population dynamics. Thus, the phenotypic composition of an evolving population may affect the direction of evolution (frequency-dependent selection, see e.g. Heino et al. 1998).

If the invasion fitness exponent is positive the mutant will invade the resident population and may replace it. If the exponent is negative the mutant will go extinct. The success of such invasions can be visualised by pairwise invasibility plots (Kisdi and Meszena 1993, Geritz et al. 1997). These plots show the sign of the invasion fitness exponents for different pairs of resident and mutant phenotypes and indicate the direction of evolution and potential evolutionary endpoints. Unfortunately, it is often impossible to calculate the invasion fitness exponents analytically and simulation models are required to find the phenotypes which can invade a given resident population (Doebeli and Ruxton 1997). This is especially true if one considers the evolution of dispersal in temporally fluctuating environments. Furthermore, if the population is structured, for example by stage or by age, in this case $N(t)$ and $\mathrm{R}(\mathrm{E}(\mathrm{t}))$ are the state vector and projection matrix, respectively. Then the invasion fitness exponent is the dominant Lyapunov exponent of the matrix sequence $\mathrm{R}(\mathrm{E}(\mathrm{t})), \mathrm{t}=0,1$, 2, ... (Tuljapurkar 1990, Metz et al. 1992, Geritz et al. 1997). The same holds if the population is spatially structured. However, for a basic understanding it is sufficient to retain the general level of eq. (1) and to approach the evolution of age specific dispersal in a spatially structured population as described below.

\section{Dispersal}

A dispersing phenotype which wants to invade in an environment averages arithmetically over the realised fitness values $\mathrm{R}^{\mathrm{h}}\left(\mathrm{E}^{\mathrm{h}}(\mathrm{t})\right)$ in the different habitats $\mathrm{h}$ within this environment (Metz et al. 1983, Holt and McPeek 1996):

$$
\mathrm{R}(\mathrm{E}(\mathrm{t}))=\sum_{\mathrm{h}} \mathrm{p}_{\mathrm{h}}(\mathrm{t}) \mathrm{R}^{\mathrm{h}}\left(\mathrm{E}^{\mathrm{h}}(\mathrm{t})\right)
$$

The weights $\mathrm{p}_{\mathrm{h}}(\mathrm{t})=\mathrm{N}_{\mathrm{h}}(\mathrm{t}) / \sum_{h} \mathrm{~N}_{\mathrm{h}}(\mathrm{t})$ describe the fraction of the total population of the mutant phenotype in habitat $h$. Thus, the weights depend on the attributes of the applied dispersal strategy of the mutant phenotype like the magnitude of dispersal, the mode of dispersal or the mortality during dispersal (note that in the latter case the weights include a factor considering the decrease of total population size after dispersal; Metz et al. 1983). In general the weights vary with time as a result of local reproduction and dispersal in a spatially heterogeneous and temporally varying environment. In connection with eq. (1) it becomes clear why a dispersing phenotype may have an advantage compared to a nondispersing one: due to the arithmetic averaging over the realised fitness values $\mathrm{R}^{\mathrm{h}}\left(\mathrm{E}^{\mathrm{h}}(\mathrm{t})\right)$ in the habitats $h$, a dispersing phenotype can compensate for bad local conditions at time t. This increases the geometric average of the realised fitness values over time (eq. (1)). In contrast, a nondispersing phenotype is confined to one patch only (at least in the long-term) and thus only equation (1) applies. How well the arithmetic averaging works determines the ability of a certain dispersal strategy to invade a resident population and thus determines its success in the evolutionary competition.

The effect of the arithmetic averaging in eq. (2) has three aspects. Firstly, the arithmetic average depends on the spatial and temporal variance in the fitness values $R^{\mathrm{h}}(\mathrm{E}$ $\mathrm{h}(\mathrm{t}))$. Such a variance can be generated by temporal fluctuations of the abiotic environment or by complex (chaotic) local population dynamics of the resident population (biotic environment). Consequently, spatially uncorrelated temporal variability of the environment selects for high dispersal (e.g. Holt and McPeek 1996). Secondly, the arithmetic average in eq. (2) depends on the weights $\mathrm{p}_{\mathrm{h}}(\mathrm{t})$. Imagine that there are both large (=good) and small (=bad) habitats within a spatially structured population. Then any nonzero dispersal rate would take on average more individuals from good (large) to bad (small) habitats. Therefore, spatial heterogeneity of the environment selects against dispersal (Hastings 1983, Levin et al. 1984, Johnson and Gaines 1990, Olivieri et al. 1995). In a spatially heterogeneous and temporally varying environment the degree of spatial heterogeneity compared to that of temporal variability influences the evolution of dispersal and often leads to a coexistence of phenotypes with low and high dispersal rates (e.g. McPeek and Holt 1992). Note that exceptions to these general rules may appear by conditional dispersal strategies, (e.g. habitat or density dependent dispersal; McPeek and Holt 1992, Johst and Brandl 1997a, b) or by demographic stochasticity (Travis and Dytham 1998). Thirdly, the number of available habitats is important. A large number of habitats can make the arithmetic averaging in eq. (2) more efficient by compensating for several low local fitness values (Johst et al. 1999). 


\section{Age specific dispersal}

How does the age of the dispersers fit into this framework? As explained above, the weights $\mathrm{p}_{\mathrm{h}}(\mathrm{t})$ of the arithmetic average given in eq. (2) depend on the population size of the mutant phenotype $\mathrm{N}_{h}(t)$ in habitat $h$ and thus on the overall magnitude of dispersal of the mutant phenotype. This magnitude is determined by the number of individuals leaving the habitat $\mathrm{h}$ during dispersal which is the product of the dispersal probability $\mathrm{m}$ and the number of potential dispersers $\mathrm{N}_{\mathrm{h}}(\mathrm{t})$ within the habitat $\mathrm{h}$ (Johst and Brandl 1999). Note that the number of immigrants from the other habitats is influenced by this product as well. Consequently, with age specific dispersal the overall magnitude of dispersal and thus the weights $\mathrm{p}_{\mathrm{h}}(\mathrm{t})$ of a mutant phenotype depend on both the age specific dispersal rates and the age specific number of potential dispersers. Therefore, the weights $\mathrm{p}_{\mathrm{h}}(\mathrm{t})$ depend on the commonness (or rarity) of the dispersing age classes. Let us consider the evolution of natal versus breeding dispersal: a mutant phenotype with a certain probability of juveniles to disperse and a mutant phenotype with the same probability of adults to disperse differ in their magnitude of dispersal according to the number of juveniles compared to the number of adults within the population. Thus, in an evolutionary context the age structure influences the efficiency of dispersal of a certain age class and can drive the evolution to age specific dispersal strategies.

Of course juveniles may disperse because they are inferior in the competition for breeding sites (Greenwood and Harvey 1982) or because prereproductive juveniles have no fecundity costs due to dispersal (Morris 1982). Furthermore, inbreeding avoidance may foster the evolution of natal dispersal (Johnson and Gaines 1990). However, even without these well-known advantages natal dispersal may evolve simply because of the influence of the age structure on the arithmetic averaging process due to dispersal. This influences the invasion fitness exponent and thus the evolutionary outcome of the competition between age specific dispersal strategies.

The above reflections show that purely demographic factors such as the distribution of the individuals across age classes or across life history stages can be responsible for the evolution of age or stage specific dispersal. Consequently, considering the commonness or rarity of the corresponding age classes may help field biologists to generate novel hypotheses for the observed plethora of age specific dispersal in nature.

\section{References}

Cockburn, A. 1992. Habitat heterogeneity and dispersal: environmental and genetic patchiness. - In: Stenseth, N. C. and Lidicker, W. Z (eds), Animal dispersal. Chapman and Hall, pp. 65-95.

Dieckmann, U. 1997. Can adaptive dynamics invade? - Trends Ecol. Evol. 12: 128-131.

Doebeli, M. and Ruxton, G. D. 1997. Evolution of dispersal rates in metapopulation models: branching and cyclic dynamics in phenotype space. - Evolution 51: 1730-1741.

Geritz, S. A. H. et al. 1997. Dynamics of adaptation and evolutionary branching. - Phys. Rev. Lett. 78: 2024-2027.

Greenwood, P. J. and Harvey, P. H. 1982. The natal and breeding dispersal of birds. - Annu. Rev. Ecol. Syst. 13: 1-21.

Hastings, A. 1983. Can spatial variation alone lead to selection for dispersal? - Theor. Popul. Biol. 24: 244-251.

Heino, M., Metz, J. A. J. and Kaitala, V. 1998. The enigma of frequency-dependent selection. - Trends Ecol. Evol. 13: 367-370.

Holt, R. D. and McPeek, M. A. 1996. Chaotic population dynamics favors the evolution of dispersal. - Am. Nat. 148: 709-718.

Johnson, M. L. and Gaines, M. S. 1990. Evolution of dispersal: theoretical models and empirical tests using birds and mammals. - Annu. Rev. Ecol. Syst. 21: 449-480.

Johst, K. and Brandl, R. 1997a. Evolution of dispersal: the importance of the temporal order of dispersal and reproduction. - Proc. R. Soc. Lond. B 264: 24-30.

Johst, K. and Brandl, R. 1997b. The effect of dispersal on local population dynamics. - Ecol. Model. 104: 87-101.

Johst, K. and Brandl, R. 1999. Natal versus breeding dispersal: evolution in a model system. - Evol. Ecol. Res. 1: 911-921.

Johst, K., Doebeli, M. and Brandl, R. 1999. Evolution of complex dynamics in spatially structured populations. - Proc. R. Soc. Lond. B 266: 1147-1154.

Kisdi, E. and Meszena, G. 1993. Density-dependent life history evolution in fluctuating environments. - In: Yoshimura, J. and Clark, C. W. (eds), Adaptions in stochastic environments. Springer, pp. 26-62.

Levin, S. A., Cohen, D. and Hastings, A. 1984. Dispersal in patchy environments. - Theor. Popul. Biol. 26: 165-191.

McPeek, M. A. and Holt, R. D. 1992. The evolution of dispersal in spatially and temporally varying environments. - Am. Nat. 140: 1010-1027.

Metz, J. A. J., deJong, T. J. and Klinkhamer, P. G. L. 1983. What are the advantages of dispersing; a paper by Kuno explained and extended. - Oecologia 57: 166-169.

Metz, J. A. J., Nisbet, R. M. and Geritz, S. A. H. 1992. How should we define fitness for general ecological scenarios? Trends Ecol. Evol. 7: 198-202.

Metz, J. A. J. et al. 1996. Adaptive dynamics: a geometrical study of the consequences of nearly faithful reproduction. - In: van Strien, S. J. and Verduyn Lunel, S. M. (eds), Stochastic and spatial structures of dynamical systems. North Holland, pp. 183-231.

Morris, D. W. 1982. Age-specific dispersal strategies in iteroparous species: who leaves when? - Evol. Theor. 6: 53-65.

Olivieri, I., Michalakis, Y. and Gouyon, P. 1995. Metapopulation genetics and the evolution of dispersal. - Am. Nat. 146: 202-227. 
Rand, D. A., Wilson, H. B. and McGlade, J. M. 1994. Dynamics and evolution: evolutionary stable attractors, invasion exponents and phenotype dynamics. - Philos. Trans. R. Soc. Lond. B 343: 261-283.

Travis, J. M. J. and Dytham, C. 1998. The evolution of dispersal in a metapopulation: a spatially explicit, individual-based model. - Proc. R. Soc. Lond. B 265: 17-23.
Tuljapurkar, S. 1990. Population dynamics in changing environments. - Springer. 